\title{
On a connection between formulas about q-gamma functions
}

Wolfram Koepf, Predrag M. Rajković, Sladjana D. Marinković

To cite this article: Wolfram Koepf, Predrag M. Rajković, Sladjana D. Marinković (2016) On a connection between formulas about $q$-gamma functions, Journal of Nonlinear Mathematical Physics 23:3, 343-350, DOI:

https://doi.org/10.1080/14029251.2016.1199496

To link to this article: https://doi.org/10.1080/14029251.2016.1199496

Published online: 04 January 2021 


\title{
On a connection between formulas about $q$-gamma functions
}

\author{
Wolfram Koepf \\ Department of Mathematics and Computer Science, University of Kassel \\ Heinrich-Plett-Str. 40, 34132 Kassel, Germany \\ koepf@mathematik.uni-kassel.de \\ Predrag M. Rajković \\ Department of Mathematics, Faculty of Mechanical Engineering, University of Niš \\ A. Medvedeva 14, 18000 Niš, Serbia \\ pedja.rajk@yahoo.com \\ Sladjana D. Marinković \\ Department of Mathematics, Faculty of Electronic Engineering, University of Niš \\ A. Medvedeva 14, 18000 Niš, Serbia \\ sladjana.marinkovic@elfak.ni.ac.rs
}

Received 8 April 2016

Accepted 13 April 2016

\begin{abstract}
In this short communication, we want to pay attention to a few wrong formulas which are unfortunately cited and used in a dozen papers afterwards. We prove that the provided relations and asymptotic expansion about the $q$-gamma function are not correct. This is illustrated by numerous concrete counterexamples. The error came from the wrong assumption about the existence of a parameter which does not depend on anything. Here, we apply a similar procedure and derive a correct formula for the $q$-gamma function.
\end{abstract}

Keywords: q-Gamma function; asymptotic expansion; boundary functions.

2000 Mathematics Subject Classification: 33D05, 11A67

\section{Introduction}

Since J. Thomae (1869) and F. H. Jackson (1904) defined the $q$-gamma function, it plays an important role in the theory of the basic hypergeometric series [5] and its applications [8]. Its properties and different representations were discussed in numerous papers, such as in [4], [12] and [11]. A few successful algorithms for its numerical evaluation are introduced in [7] and [6] and [1]. An asymptotic expansion of the $q$-gamma function was provided in [3].

Here, we will make observations on the asymptotic expansions given in $[9,10]$.

Let $q \in[0,1)$. A $q$-number $[a]_{q}$ is

$$
[a]_{q}:=\frac{1-q^{a}}{1-q}, \quad a \in \mathbb{R} .
$$

The factorial of a positive integer number $[n]_{q}$ is given by

$$
[0]_{q} !:=1, \quad[n]_{q} !:=[n]_{q}[n-1]_{q} \cdots[1]_{q}, \quad(n \in \mathbb{N}) .
$$


An important role in $q$-calculus plays the $q$-Pochhammer symbol defined by

$$
(a ; q)_{0}=1, \quad(a ; q)_{n}=\prod_{i=0}^{n-1}\left(1-a q^{i}\right) \quad(n \in \mathbb{N} \cup\{+\infty\}),
$$

and

$$
(a ; q)_{\lambda}=\frac{(a ; q)_{\infty}}{\left(a q^{\lambda} ; q\right)_{\infty}} \quad(|q|<1, \lambda \in \mathbb{C})
$$

The $q$-gamma function

$$
\Gamma_{q}(z)=(q ; q)_{z-1}(1-q)^{1-z}=\frac{(q ; q)_{\infty}}{\left(q^{z} ; q\right)_{\infty}}(1-q)^{1-z} \quad\left(0<q<1, z \notin \mathbb{Z}^{-}\right)
$$

has the following properties:

$$
\Gamma_{q}(z+1)=[z]_{q} \Gamma_{q}(z) \quad(z \in \mathbb{C}), \quad \Gamma_{q}(n+1)=[n]_{q} ! \quad\left(n \in \mathbb{N}_{0}\right) .
$$

In particular,

$$
\lim _{q \rightarrow 1-} \Gamma_{q}(z)=\Gamma(z) .
$$

The exact $q-$ Gauss multiplication formula can be found in [5] or [4]:

$$
\Gamma_{q}(n x) \prod_{k=1}^{n-1} \Gamma_{q^{n}}\left(\frac{k}{n}\right)=[n]_{q}^{n x-1} \prod_{k=0}^{n-1} \Gamma_{q^{n}}\left(x+\frac{k}{n}\right) \quad(x>0 ; n \in \mathbb{N}) .
$$

Equivalently, substituting $z=n x$, it can be written in the form

$$
\Gamma_{q}(z) \prod_{k=1}^{n-1} \Gamma_{q^{n}}\left(\frac{k}{n}\right)=[n]_{q}^{z-1} \prod_{k=0}^{n-1} \Gamma_{q^{n}}\left(\frac{z+k}{n}\right) \quad(z>0 ; n \in \mathbb{N}) .
$$

\section{Our corrections to the paper [9]}

Starting from the definition

$$
\Gamma_{q}(x)=(q ; q)_{\infty}(1-q)^{1-x}\left(q^{x} ; q\right)_{\infty}^{-1}
$$

we can write

$$
\Gamma_{q}(x)=(q ; q)_{\infty}(1-q)^{1 / 2}(1-q)^{1 / 2-x} e^{-\log \left(q^{x} ; q\right)_{\infty}} .
$$

Hence the function $\Gamma_{q}(x)$ can be written in the form

$$
\Gamma_{q}(x)=a(q) \cdot(1-q)^{1 / 2-x} e^{\mu(x)} \quad(a(q) \in \mathbb{R}),
$$

where

$$
0<a(q)=(q ; q)_{\infty}(1-q)^{1 / 2}<1, \quad \mu(x, q)=-\log \left(q^{x} ; q\right)_{\infty} .
$$

Let

$$
\psi(x, q)=\frac{q^{x}}{(1-q)\left(1-q^{x}\right)} .
$$


From the estimate

$$
0<\mu(x, q)<\psi(x, q) \quad(0<q<1, x>0),
$$

it exists $\theta(x, q) \in(0,1)$ such that

$$
\mu(x, q)=\theta(x, q) \cdot \psi(x, q) .
$$

Therefore, relation (2.1) becomes

$$
\Gamma_{q}(x)=a(q) \cdot(1-q)^{1 / 2-x} e^{\theta(x, q) \cdot \psi(x, q)} .
$$

On the other hand, formula (1.2) can be written in the form

$$
a_{p}(q) \Gamma_{q}(x)=[p]_{q}^{x} \prod_{k=0}^{p-1} \Gamma_{q^{p}}\left(\frac{x+k}{p}\right) \quad(x>0 ; p \in \mathbb{N}),
$$

where

$$
a_{p}(q)=[p]_{q} \Gamma_{q^{p}}\left(\frac{1}{p}\right) \Gamma_{q^{p}}\left(\frac{2}{p}\right) \cdots \Gamma_{q^{p}}\left(\frac{p}{p}\right) .
$$

Substituting $q \rightarrow q^{p}$ and $x \rightarrow k / p$ into the definition (1.1) of the $q$-gamma function, we have

$$
\Gamma_{q^{p}}\left(\frac{k}{p}\right)=\frac{\left(q^{p} ; q^{p}\right)_{\infty}}{\left(q^{k} ; q^{p}\right)_{\infty}}\left(1-q^{p}\right)^{1-k / p}=\left(1-q^{p}\right)^{1-k / p} \lim _{n \rightarrow \infty} \frac{\left(q^{p} ; q^{p}\right)_{n}}{\left(q^{k} ; q^{p}\right)_{n}} .
$$

Moreover, using

$$
\prod_{k=1}^{p}\left(1-q^{p}\right)^{1-k / p}=\left(1-q^{p}\right)^{\frac{p-1}{2}}
$$

the following holds:

$$
\begin{aligned}
a_{p}(q) & =[p]_{q} \prod_{k=1}^{p} \Gamma_{q^{p}}\left(\frac{k}{p}\right)=[p]_{q} \prod_{k=1}^{p}\left(1-q^{p}\right)^{1-k / p} \lim _{n \rightarrow \infty} \frac{\left(q^{p} ; q^{p}\right)_{n}}{\left(q^{k} ; q^{p}\right)_{n}} \\
& =[p]_{q} \prod_{k=1}^{p}\left(1-q^{p}\right)^{1-k / p} \lim _{n \rightarrow \infty} \frac{\left(q^{p} ; q^{p}\right)_{n}^{p}}{\prod_{k=1}^{p}\left(q^{k} ; q^{p}\right)_{n}} \\
& =[p]_{q}\left(1-q^{p}\right)^{\frac{p-1}{2}} \lim _{n \rightarrow \infty} \frac{\left(q^{p} ; q^{p}\right)_{n}^{p}}{\prod_{k=1}^{p}\left(q^{k} ; q^{p}\right)_{n}} .
\end{aligned}
$$

The following identity is valid

$$
\prod_{k=1}^{p}\left(q^{k} ; q^{p}\right)_{n}=(q ; q)_{n p}
$$

Using estimate (2.3), we get

$$
\Gamma_{q^{p}}(n+1)=a\left(q^{p}\right) \cdot\left(1-q^{p}\right)^{-n-1 / 2} \cdot e^{\theta\left(n+1, q^{p}\right) \cdot \psi\left(n+1, q^{p}\right)}
$$

Since

$$
\frac{\left(q^{p} ; q^{p}\right)_{n}^{p}}{\left(1-q^{p}\right)^{n p}}=\Gamma_{q^{p}}^{p}(n+1)=a^{p}\left(q^{p}\right) \cdot\left(1-q^{p}\right)^{p(-1 / 2-n)} \cdot e^{p \cdot \theta\left(n+1, q^{p}\right) \cdot \psi\left(n+1, q^{p}\right)},
$$


and

$$
\frac{\prod_{k=1}^{p}\left(q^{k} ; q^{p}\right)_{n}}{(1-q)^{n p}}=\frac{(q ; q)_{n p}}{(1-q)^{n p}}=\Gamma_{q}(n p+1)=a(q) \cdot(1-q)^{-1 / 2-n p} \cdot e^{\theta(n p+1, q) \cdot \psi(n p+1, q)},
$$

we have

$$
a_{p}(q)=\frac{a^{p}\left(q^{p}\right)}{a(q)}[p]_{q}^{1 / 2} \lim _{n \rightarrow \infty} \frac{e^{p \cdot \theta\left(n+1, q^{p}\right) \cdot \psi\left(n+1, q^{p}\right)}}{e^{\theta(n p+1, q) \cdot \psi(n p+1, q)}}
$$

From

$$
\lim _{n \rightarrow \infty} \psi\left(n+1, q^{p}\right)=\lim _{n \rightarrow \infty} \psi(n p+1, q)=0 \quad(0<q<1 ; p \in \mathbb{N})
$$

we find

$$
a_{p}(q)=[p]_{q}^{1 / 2} \frac{a^{p}\left(q^{p}\right)}{a(q)} .
$$

In that manner, the parameter $a_{p}(q)$ from formula (2.4) is expressed via the parameter $a(q)$ from formula (2.3).

\section{Faults in paper [9]}

In the very beginning of this section, we wish to express our opinion that in [9] an excellent approach was exposed, but a few mistakes were made in its realization. So, we have decided to refer to them. In [9], the author has supposed that $\Gamma_{q}(x)$ for $0<q<1 ; x>0$, can be written in the form

$$
\Gamma_{q}(x)=a \cdot(1-q)^{1 / 2-x} e^{\mu(x)} \quad(a \in \mathbb{R}),
$$

where

$$
\mu(x)=-\log \left(q^{x} ; q\right)_{\infty}>0 .
$$

His efforts in looking for $\mu(x)$ we shortened a lot by starting from the definition of $\Gamma_{q}(x)$. From the fact that

$$
0<\mu(x)<\frac{q^{x}}{(1-q)\left(1-q^{x}\right)}
$$

and

$$
(1-q)\left(1-q^{x}\right)=1-q-q^{x}+q^{x+1}>1-q-q^{x},
$$

the author in [9] concluded wrongly that

$$
0<\mu(x)<\frac{q^{x}}{(1-q)-q^{x}} .
$$

But, expression $1-q-q^{x}$ is not positive for all $q \in(0,1)$ and $x>0$. Indeed,

$$
1-q-q^{x} \leq 0 \Leftrightarrow 1-q \leq q^{x} \Leftrightarrow x \cdot \log q \geq \log (1-q) \Leftrightarrow x \leq \frac{\log (1-q)}{\log q} .
$$

Example 3.1. We examined the sign changes of the function $h_{q}(x) \equiv 1-q-q^{x}$ for different $q$ and $x$. Notice that $x \rightarrow+\infty$ if $q \rightarrow 1^{-}$. 
Table 1. Unique real zero of the function $h_{q}(x)$ and the sign changes for random values of $q$ and $x$

\begin{tabular}{cr}
\hline$q$ & $x: 1-q-q^{x}=0$ \\
\hline 0.1 & 0.045758 \\
0.3 & 0.296248 \\
0.5 & 1.0000 \\
0.7 & 3.37555 \\
0.9 & 21.8543 \\
\hline
\end{tabular}

\begin{tabular}{crr}
\hline$x$ & $q$ & $1-q-q^{x}$ \\
\hline 1.10500 & 0.592727 & -0.15378 \\
2.27287 & 0.752038 & -0.275286 \\
6.47584 & 0.816692 & -0.0861563 \\
43.2362 & 0.946066 & -0.0370453 \\
60.1635 & 0.954814 & -0.0167368 \\
\hline
\end{tabular}

This estimate should be written in the from

$$
0<\mu(x)<\frac{q^{x}}{(1-q)-q^{x}} \quad\left(0<q<1 ; x>\frac{\log (1-q)}{\log q}\right) .
$$

Furthermore, from the estimate

$$
0<\mu(x)<\frac{q^{x}}{(1-q)-q^{x}}
$$

the author in [9] concluded wrongly that

$$
\mu(x)=\frac{\theta q^{x}}{(1-q)-q^{x}},
$$

where $\theta$ is a number independent of $x$ between 0 and 1 .

Example 3.2. We find counterexamples which show that $\theta$ depends on $x$ and $q$. In the first table, we fixed $q=0.9$ and take a few random values for $x$. In another we changed the rule of variables.

Table 2. The dependence of parameter $\theta$ from $x$ and $q$

\begin{tabular}{ccr}
\hline$x$ & $q$ & \multicolumn{1}{c}{$\theta$} \\
\hline 3.78377 & 0.9 & -7.27980 \\
13.2554 & 0.9 & -1.58344 \\
20.6473 & 0.9 & -0.139893 \\
25.7471 & 0.9 & 0.342512 \\
32.2948 & 0.9 & 0.673069 \\
43.8850 & 0.9 & 0.904181 \\
\hline
\end{tabular}

\begin{tabular}{crr}
\hline$x$ & $q$ & $\theta$ \\
\hline 10.5 & 0.063920 & 1.00000 \\
10.5 & 0.234682 & 1.00000 \\
10.5 & 0.494904 & 0.99898 \\
10.5 & 0.618621 & 0.98504 \\
10.5 & 0.806515 & 0.473541 \\
10.5 & 0.915828 & -4.19862 \\
\hline
\end{tabular}

In continuation, the author in [9] got the wrong formulas (2.21)-(2.27). He concluded that

$$
a_{p}=\sqrt{[2]_{q}} \Gamma_{q^{2}}(1 / 2)
$$

and

$$
\Gamma_{q}(x)=\sqrt{[2]_{q}} \Gamma_{q^{2}}(1 / 2)(1-q)^{1 / 2-x} e^{\theta \frac{q^{x}}{(1-q)-q^{x}}} \quad(0<\theta<1) .
$$

The following wrong version of the $q$-Gauss multiplication formula was provided

$$
[n]_{q}^{1 / 2-x}[2]_{q}^{(n-1) / 2} \Gamma_{q^{2}}^{n-1}(1 / 2) \Gamma_{q}(x)=\prod_{k=0}^{n-1} \Gamma_{q^{n}}\left(\frac{x+k}{n}\right) \quad(x>0 ; n \in \mathbb{N}) .
$$


In a special case, for $n=2$, it agrees with the exact $q$-Legendre relation. Also, when $q \rightarrow 1$, it reduces to well-known formulas for the gamma function.

\section{Bounds of the $q$-gamma function}

Let

$$
g(x)=\ln \Gamma_{q}(x)
$$

Since

$$
g(x+1)=\ln \Gamma_{q}(x+1)=\ln \left([x]_{q} \Gamma_{q}(x)\right)=\ln [x]_{q}+g(x),
$$

by induction, we get

$$
g(x+n)=\sum_{k=0}^{n-1} \ln [x+k]_{q}+g(x) \quad(n \in \mathbb{N}) .
$$

In the paper [2] it was proven that $g(x)$ is a convex function.

Lemma 4.1. If $x \in(0,1)$ and $n \in \mathbb{N}$, then

$$
g(n)+x \ln [x+n-1]_{q} \leq g(x+n) \leq(1-x) g(n)+x g(n+1)
$$

Proof. Since

$$
x+n=(1-x) n+x(n+1),
$$

we can write

$$
g(x+n)=g((1-x) n+x(n+1)) \leq(1-x) g(n)+x g(n+1) .
$$

Let us find a lower bound for $\Gamma_{q}(x)$. Since

$$
n=(1-x)(x+n)+x(x+n-1),
$$

and because of the convexity of the function $g(x)$, we have

$$
g(n) \leq(1-x) g(x+n)+x g(x+n-1) .
$$

Applying (4.1), for $x \rightarrow x+n-1$, we can write

$$
g(x+n)=\ln [x+n-1]_{q}+g(x+n-1),
$$

wherefrom

$$
g(n) \leq(1-x) g(x+n)+x\left(g(x+n)-\ln [x+n-1]_{q}\right)=g(x+n)-x \ln [x+n-1]_{q},
$$

i.e.,

$$
g(n)+x \ln [x+n-1]_{q} \leq g(x+n) .
$$

Theorem 4.1. The following bounds are valid:

$$
[n-1]_{q} ![n-1+x]_{q}^{x} \leq \Gamma_{q}(n+x) \leq[n-1]_{q} ![n]_{q}^{x} \quad(n \in \mathbb{N} ; 0 \leq x<1) .
$$


Proof. According to the upper bound for $g(x)$, we get e. g.

$$
\ln \Gamma_{q}(x+n) \leq(1-x) \ln \Gamma_{q}(n)+x \ln \Gamma_{q}(n+1) .
$$

Since the real logarithm is an increasing and continuous function, we have

$$
\Gamma_{q}(x+n) \leq\left([n-1]_{q} !\right)^{1-x}\left([n]_{q} !\right)^{x},
$$

wherefrom

$$
\Gamma_{q}(x+n) \leq[n-1]_{q} ![n]_{q}^{x} .
$$

According to the lower bound for $g(x)$, we get

$$
\ln \Gamma_{q}(n)+x \ln [x+n-1]_{q} \leq \ln \Gamma_{q}(x+n),
$$

i.e.,

$$
\Gamma_{q}(n)[n+x-1]_{q}^{x} \leq \Gamma_{q}(n+x)
$$

Theorem 4.2.

$$
[n-(1-x)]_{q} \leq\left(\frac{\Gamma_{q}(n+x)}{[n-1]_{q} !}\right)^{1 / x} \leq[n]_{q} \quad\left(n \in \mathbb{N}_{0} ; 0 \leq x<1\right) .
$$
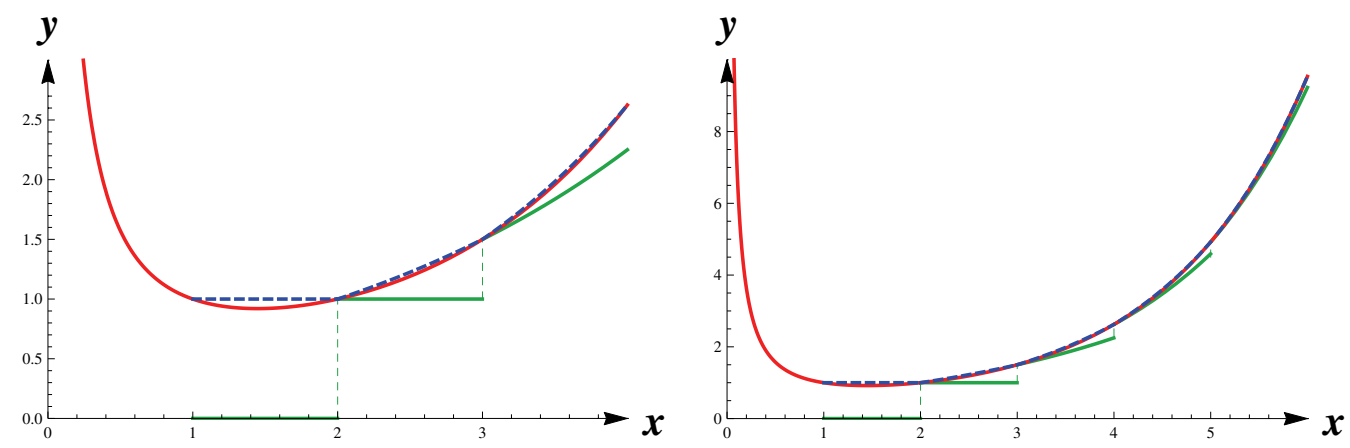

Fig. 1. $\Gamma_{q}(x)$ and its boundary functions (green and blue) for $q=0.5$.

Theorem 4.3. For any $n \in \mathbb{N}$ and $x \in(0,1)$ there exists $\theta=\theta(n, x, q) \in(0,1)$ such that

$$
\Gamma_{q}(n+x)=[n-1]_{q} ![n-\theta(1-x)]_{q}^{x} .
$$

Introducing $y=n+x\left(n \in \mathbb{N}_{0} ; 0 \leq x<1\right)$ and denoting $n=\lfloor y\rfloor$, we can write

$$
[\lfloor y\rfloor-1]_{q} ![y-1]_{q}^{y-\lfloor y\rfloor} \leq \Gamma_{q}(y) \leq[\lfloor y\rfloor-1]_{q} ![\lfloor y\rfloor]_{q}^{y-\lfloor y\rfloor} \quad(y>1) .
$$

Theorem 4.4. For any $y \in(1,+\infty) \backslash \mathbb{N}$, there exists $\theta=\theta(y, q) \in(0,1)$ such that

$$
\Gamma_{q}(y)=[\lfloor y\rfloor-1]_{q} ![\lfloor y\rfloor-\theta(1-(y-\lfloor y\rfloor))]_{q}^{y-\lfloor y\rfloor} .
$$



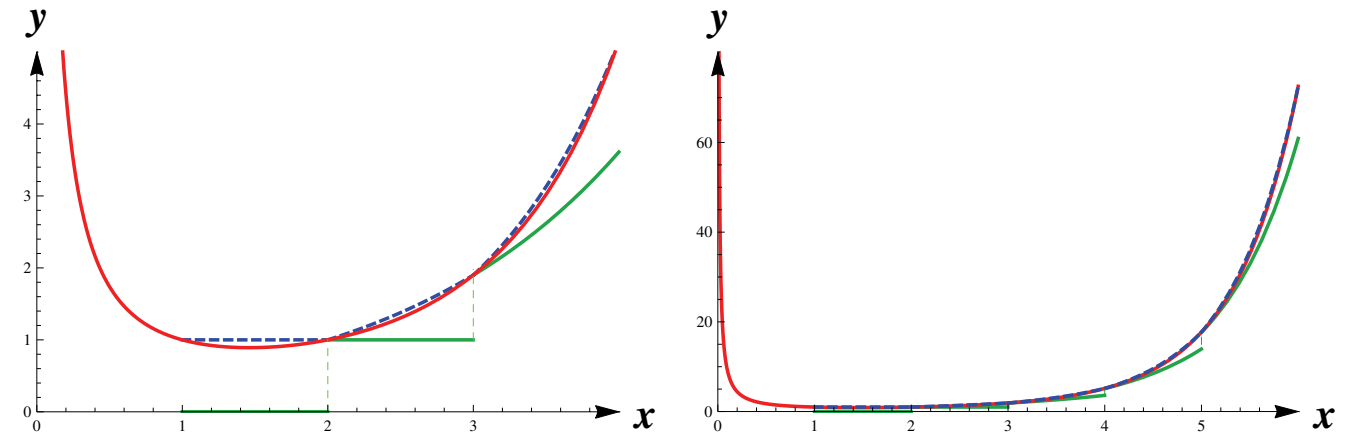

Fig. 2. $\Gamma_{q}(x)$ and its boundary functions (green and blue) for $q=0.9$.

Example 4.1. For $y=15.5$ and $q=0.1(0.1) 0.9$, we have got the following values for $\theta$ :

$$
\hat{\theta}=\{0.9851,0.4021,0.4259,0.4432,0.4569,0.4681,0.47762,0.4855,0.4917\} .
$$

Also, for $q=0.5$ and $y=2.31(2) 22.31$, we have got $\theta \in(0.4468,0.4623)$.

\section{Acknowledgement}

This paper is supported by the Ministry of Science and Technological Development of the Republic Serbia, projects No 174011.

\section{References}

[1] G. Allasia, F. Bonardo, On the Numerical Evaluation of Two Infinite Products, Mathematics of Computation 35 No. 151 (1980) 917-931.

[2] R. Askey, The $q$-gamma and $q$-beta functions, Appl. Anal. 8 (1978) 125-141.

[3] A.B.O. Daalhuis, Asymptotic Expansions for $q$-Gamma, $q$-Exponential, and $q$-Bessel Functions, Journal of Math. Analysis and Applications 186 (1994) 896-1994.

[4] I. Ege, E. Yyldyrym, Some generalized equalities for the $q$-gamma function, Filomat 26 No. 6 (2012) 1227-1232.

[5] G. Gasper, M. Rahman, Basic Hypergeometric Series, 2nd Ed, Encyclopedia of Math. and its Appl, 96 (Cambridge University Press, Cambridge 2004).

[6] B. Gabutti, G. Allasia, Evaluation of $q$-gamma function and q-analogues by iterative algorithms, Numer Algor 49 (2008) 159-168.

[7] L. Gateschi, Procedimenti iterativi per il calcolo numerico di due prodotti infiniti, Rend. Sem. Mat. Univ. Politec. Torino 29 (1969/70) 187-201.

[8] T.H. Koornwinder, Special functions and q-commuting variables, in Special Functions, $q$-Series and Related Topics, M.E.H. Ismail, D.R. Masson and M. Rahman (eds.), Fields Institute Communications 14, American Mathematical Society (1997) 131-166.

[9] M. Mansour, An asymptotic expansion of the q-gamma function $\Gamma_{q}(x)$, Journal of Nonlinear Mathematical Physics 13 No. 4 (2006) 479-483.

[10] M. Mansour, A Family of Sequences Related to an Asymptotic Expansion of the q-gamma Function, Int. Journal of Math. Analysis 3 No. 23 (2009) 1131-1137.

[11] M. Mansour, On the functional equations of the q-Gamma function, Aequationes Mathematicae 89 (2015) 1041-1050.

[12] A. Sole, V.G. Kac, On integral representations of $q$-gamma and $q$-beta functions, Rend. Mat. Acc. Lincei 9 (2005) 11-29. 